\title{
Investigating the application of reactive polymers in fluid loss control
}

\author{
Tony Trifunoski $^{1}$ ( Masood Mostofi ${ }^{1}$
}

Received: 6 January 2019 / Accepted: 17 August 2019 / Published online: 30 August 2019

(c) The Author(s) 2019

\begin{abstract}
Water reactive polymers are rapid setting fluids that exhibit high viscosity after being exposed to water to control severe fluid loss. In this study, the performance of a water reactive polymer is studied through a series of fluid loss experiments. Various unconsolidated and permeable formations were simulated using different sand size grades, with the change in permeability being recorded after applying the reactive polymer under different operating conditions. The results showed $62-100 \%$ permeability reductions depending on the initial value of permeability and operating conditions. In addition, the setting time of the reactive polymer was obtained at different water concentrations and temperatures by monitoring the viscosity of the reactive polymer using a Brookfield viscometer. The results indicate that a hydration time of $30 \mathrm{~min}$ is satisfactory to reach an acceptable viscosity for all hydration volumes tested. At lower hydration volumes, the viscosity increased rapidly at the same rate and reached the same maximum viscosity. As hydration volume increased, both the rate of increase and maximum viscosity were reduced.
\end{abstract}

Keywords Fluid loss $\cdot$ Drilling fluid $\cdot$ Reactive polymer $\cdot$ Polymer $\cdot$ PHPA $\cdot$ LCM $\cdot$ Fluid loss additive

\section{Introduction}

Lost circulation is the loss of drilling fluid from the borehole into a formation. It occurs when drilling into fractured, cavernous, vuggy and highly permeable formation (Hashmat et al. 2016; Al-saba Mortadha et al. 2014; Bugbee 1953) and can lead to large costs in drilling operations causing non-productive time in drilling, and in more severe cases, it can lead to well control problems (Kumar et al. 2011). The estimated costs for treating lost circulation zones and replacing lost fluids reach $\$ 800$ million globally each year (Ivan and Bruton 2003; Murchison 2006).

Fluid loss is typically controlled using lost circulation materials (LCMs), which are often selected by experience from previously drilled offset wells. Past research has shown that there is no "one size fits all" solution for lost circulation, meaning that each LCM must be thoroughly investigated to find the range of their effectiveness, which is dependent on the type and intensity of fluid mechanism present (ElSayed et al. 2007; Whitfill et al. 2007). When encountering a fluid loss situation, particulate LCMs are often used as

Tony Trifunoski

tony.trifunoski@outlook.com

1 Curtin University, Bentley, Australia the first attempt at curing the losses without having to apply more complicated and costlier treatment methods (Al-saba Mortadha et al. 2014). The particulate LCM can either be implemented as a concentrated pill treatment targeting a problem zone or added to the drilling fluid prior to drilling through a fluid loss zone to prevent significant losses. However, conventional particulate-based LCMs have inconsistent performance in severe cases of fluid loss which can be linked to the changing fracture sizes of natural fractures, making it difficult to find an optimal size distribution (Savari et al. 2013; Droger et al. 2014; Caughron et al. 2002).

In the cases where particulate LCMs are ineffective as a preventative solution, reactive solutions will be used as remedial fluid loss control. These fluids can be in the form of non-mud system, such as cement, chemically crosslinked pills and reactive polymers. For the reactive polymer to be effective, they must be sufficiently permanent at high temperatures and pressures, must withstand large pressure differentials, be easily applied in the field without special equipment and be solid free to allow for deep penetration into the formation (Vidick et al. 1988). The downside to these special remedial solutions is that many require time for preparation, curing and setting and tripping out downhole equipment. 
Reactive fluids are an example of a remedial system suitable for curing severe lost circulation (Al-saba Mortadha et al. 2014; Caughron et al. 2002). They are composed of cross-linking polymers that form a highly viscous fluid once activated by a cross-linking agent. Reactive fluids can be injected into the formation as a pill where they can be activated by bottom-hole conditions and/or with the addition of an activator. Once activated in the formation, they form a pressure-resilient seal in fractured and porous formations, thus effectively reducing the permeability. Past research has shown that cross-linking polymers are effective for a wide range of formations since they are not limited by pore throat size or the fracture aperture (Caughron et al. 2002). Previously, cross-linked polymers have been used to completely seal water-producing zones from both fractures and matrix, which forms the idea of their potential use as an LCM (Hashmat et al. 2016). In this paper, the reactive fluid "AMC Stick-Up" will be investigated. Stick-up is a crosslinked partially hydrolysed polyacrylamide (PHPA) which is activated by water hydration, which causes a significant increase in its viscosity.

This paper presents the findings from three methodologies used to investigate the possible application of the water reactive polymer. Firstly, the polymer's effectiveness at reducing the permeability of various unconsolidated sand formations was tested using a custom-built fluid loss simulator. Secondly, the effect of various water/polymer ratios was investigated by continually monitoring the viscosity of the mixture to observe changes in rheology. Finally, the increase in the formation strength and thus improvement in wellbore stability as a result of implementing the reactive polymer were studied by completing the scratch testing.

\section{Experimental programme}

\section{Fluid loss simulator}

A fluid loss simulator was used to measure the reactive fluids' performance at reducing permeability of unconsolidated sand formations. The permeability of the sand was measured prior to and following the application of the fluid loss additive. A variety of different sand grades were used as the filter layer ranging from $0.5-1.5 \mathrm{~mm}$ to $3.2-9.6 \mathrm{~mm}$, with each

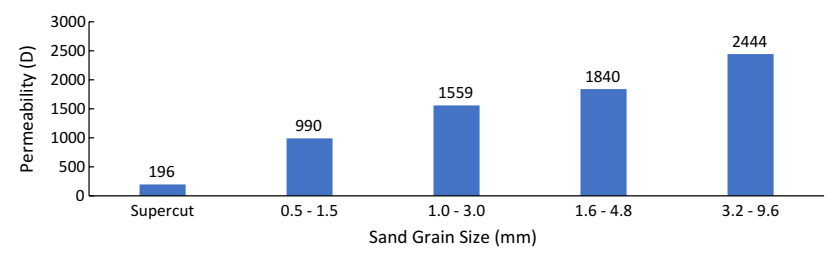

Fig. 1 Permeability of different sand grades grade simulating different permeability formations, thus benchmarking the polymers' performance over a range of permeability.

The filter loss simulator is designed similarly to the commonly used API filter press and permeability plugging apparatus (PPA) and similar experimental setup (Abdelaal et al. 2016), with pressure being applied to create a linear flow through a filter medium. The filter medium is $30 \mathrm{~cm}$ of unconsolidated sands simulating the porous formations encountered while drilling compared to using filter paper (Figs. 1 and 2).

The fluid loss simulator determines the linear permeability of the sand layer by flowing water through the sand

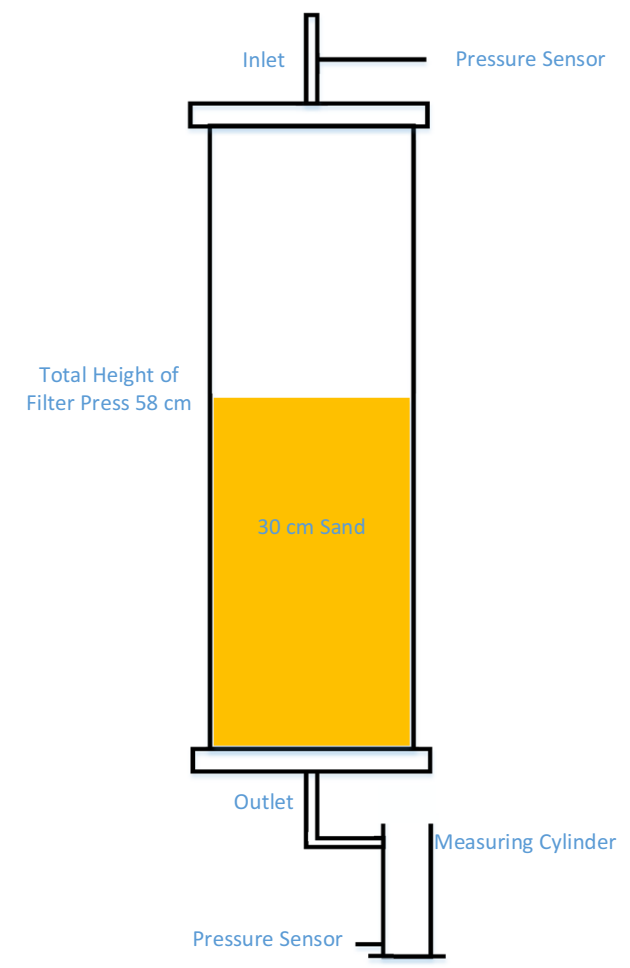

Fig. 2 Modified filter press

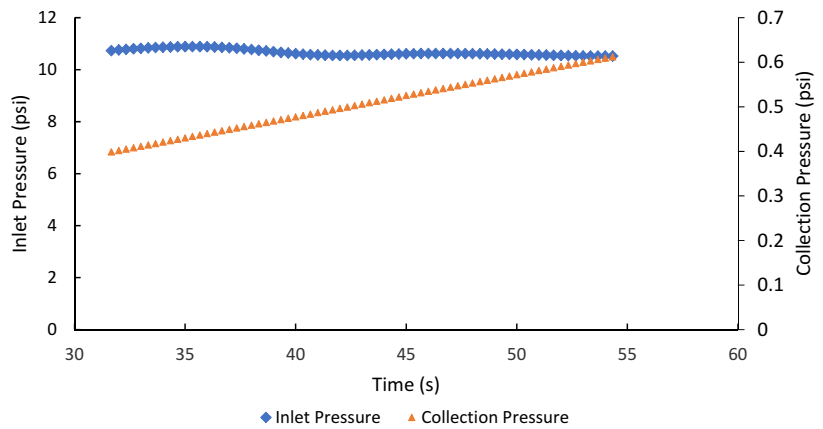

Fig. 3 Sample of pressure data 
layer while a constant inlet pressure is applied to displace the testing fluid into the simulated formation. The return fluid is collected in a 1-L graduated measuring cylinder connected to a pressure sensor. Figure 3 shows sample data recorded during the draining phase. The pressure readings from the collecting cylinder are then calibrated with $100 \mathrm{~mL}$ increments in return fluid to measure the flow rate during the experiment (Fig. 4). Based on the volume of collected fluid, the height change in the test tube is calculated, and therefore, the contribution of hydrostatic head from the fluid is included in the permeability calculation. Figure 5 shows sample data of the variation in permeability and flow rate during a test.

After the identification of the initial permeability, the process was repeated following the application of $250 \mathrm{~mL}$ of the reactive polymer in the formation.

Each sand grade was tested three times with each test having at least two repeat trials to find averages for initial permeability and post-treatment permeability. The repeat tests verified that the methodology was precise with the data range.

\section{Rheology}

The effects of hydration time and hydration volume were investigated using a Brookfield DV3T rheometer to form an understanding of the possible variations in the performance of the reactive polymer. Tests were conducted at $0.01 \mathrm{RPM}$ and $0.02 \mathrm{RPM}$ for $20 \mathrm{~min}$ or until viscosity reached the machine measurement limit. Tests were repeated with hydration volume being increased from a 1:1 ratio to a final ratio of 1:11 (polymer/water). Tests were also performed at increased temperatures to observe any changes in effectiveness.

The samples were firstly prepared by mixing water and the reactive polymer to create a well-mixed $500-\mathrm{mL}$ sample. Although the thorough premixing leads to different

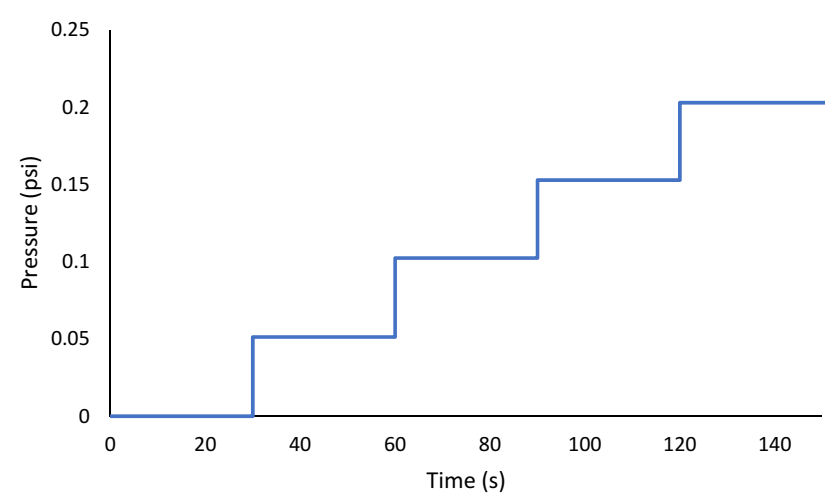

Fig. 4 Calibration step data (averages shown)

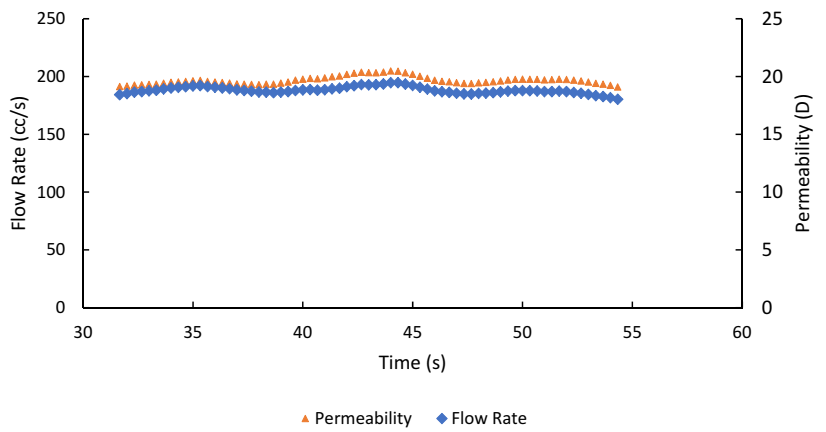

Fig. 5 Flow rates effect on recorded permeability

starting viscosities, as shown in Fig. 9, it was required to ensure the polymer was evenly hydrated.

\section{Formation strengthening}

A scratch test was performed to investigate the increase in strength of the formation after being injected with the reactive polymer. To complete the scratch test, the reactive polymer was injected into unconsolidated sand with a high rheology water/polymer ratio of 1:1. Using unconsolidated sands meant the results recorded were solely from the polymer, since the formation strength of the loose sands is minuscule. The experiments were conducted using a 10-mm sharp rectangular cutter (Fig. 6). The rock strength was calculated over the length of the sample by dividing the shear force (Fs) by the area of the cutter (Ac).

\section{Experimental results}

\section{Fluid loss control performance}

The permeability reduction following the application of the reactive polymer is shown in Figs. 7 and 8. The results show that permeability was effectively reduced; however, it

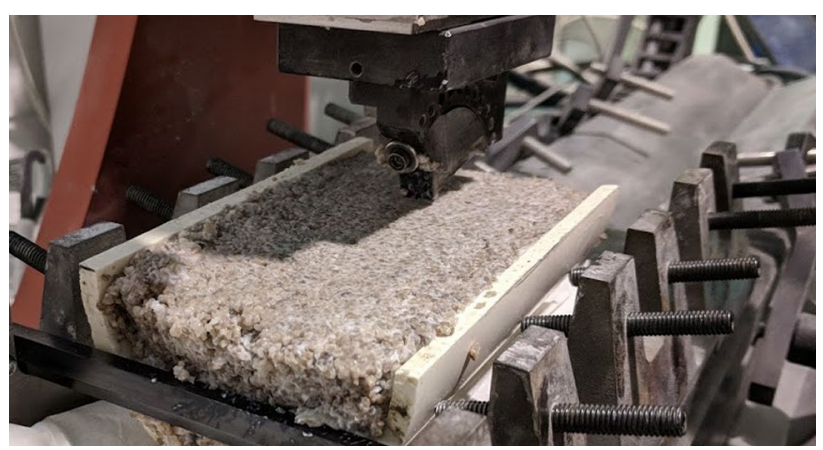

Fig. 6 Scratch test performed on 2-mm sand 


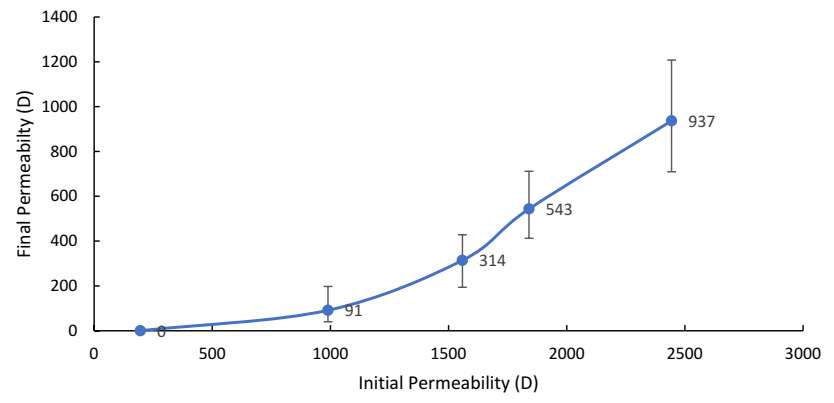

Fig. 7 Final permeability following the application of polymer

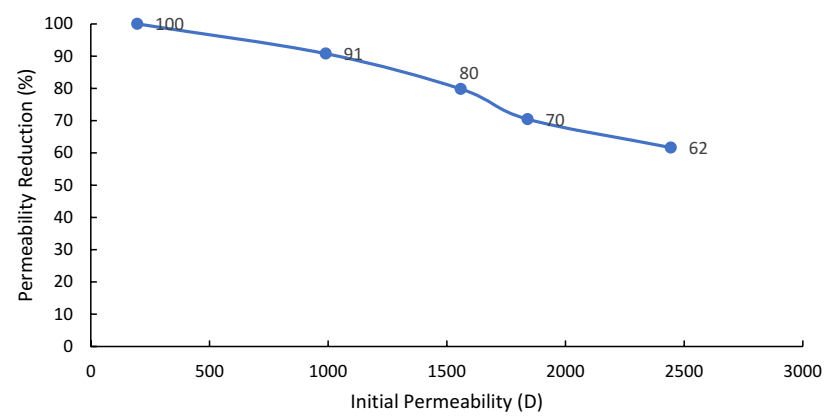

Fig. 8 Percent reduction in permeability following the application of stick-up

is evident that as initial permeability increases, the relative permeability reduction decreases; this is seen in the decreasing percentage reduction in Fig. 8.

To ensure all testing was completed under the same conditions, an inlet pressure of 10 psi was used to ensure sand compaction, and thus, matrix permeability was the same for all tests. However, due to uncontrollable effects such as grain sorting, there were some small variations in permeability between tests. The black bars in Fig. 7 represent the range of data recorded over the testing completed (Fig. 9).

\section{Curing and contamination}

The effect of different hydration volumes on the final rheology at 0.02 RPM and 0.01 RPM is shown in Figs. 10 and 11, respectively. Initially, it can be seen that at lower hydration volumes (ratios 1:1-1:6) the viscosity increases at a similar rate until the torque limit of the machine is reached. At higher hydration volume beyond a ratio of 1:6, the viscosity initially increases with a similar rate of smaller hydration volumes; however, it reaches a measurable maximum viscosity. This leads to the conclusion that as the hydration ratio increases, the maximum viscosity of the mixture decreases. After a further increase in hydration volume to a ratio of $1: 11$, the linear increase in viscosity is not present and the maximum viscosity is significantly lower.

The results recorded under 0.01 RPM showed similar behaviour. As the hydration ratio increased, the rate of increase was consistent until reaching a certain maximum ratio, in this case $1: 10$ polymer to water.

To verify the conclusion that the reactive polymer became less effective with an increase in hydration ratio, repeat tests were completed with the fluid loss simulator. The procedure used was the same as the previous testing except for the polymer being purposely contaminated with different volumes of water prior to application. It can be seen that as the hydration ratio increased, the effectiveness of the LCMs significantly declined. Each data point is the average of three separate tests.

Testing was not complete for contaminations between 1:1 and 1:6 since the viscosity increased too rapidly and the contaminated fluid could not be displaced into the formation.

Fig. 9 Rheology at 0.02 RPM

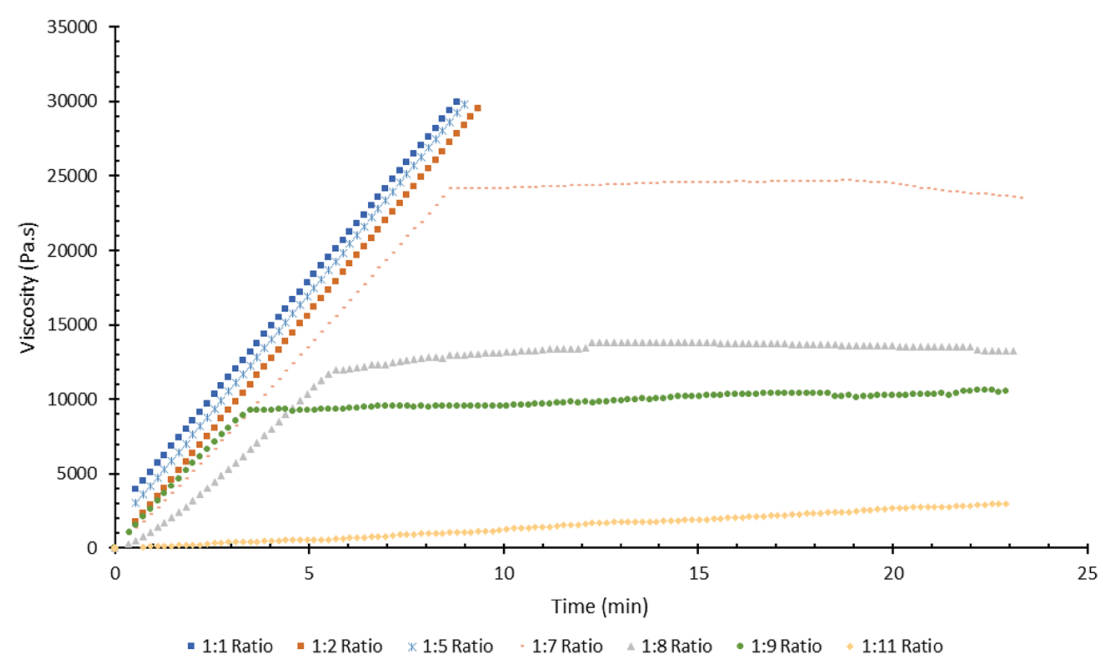


Fig. 10 Rheology of the reactive polymer at $0.01 \mathrm{RPM}$
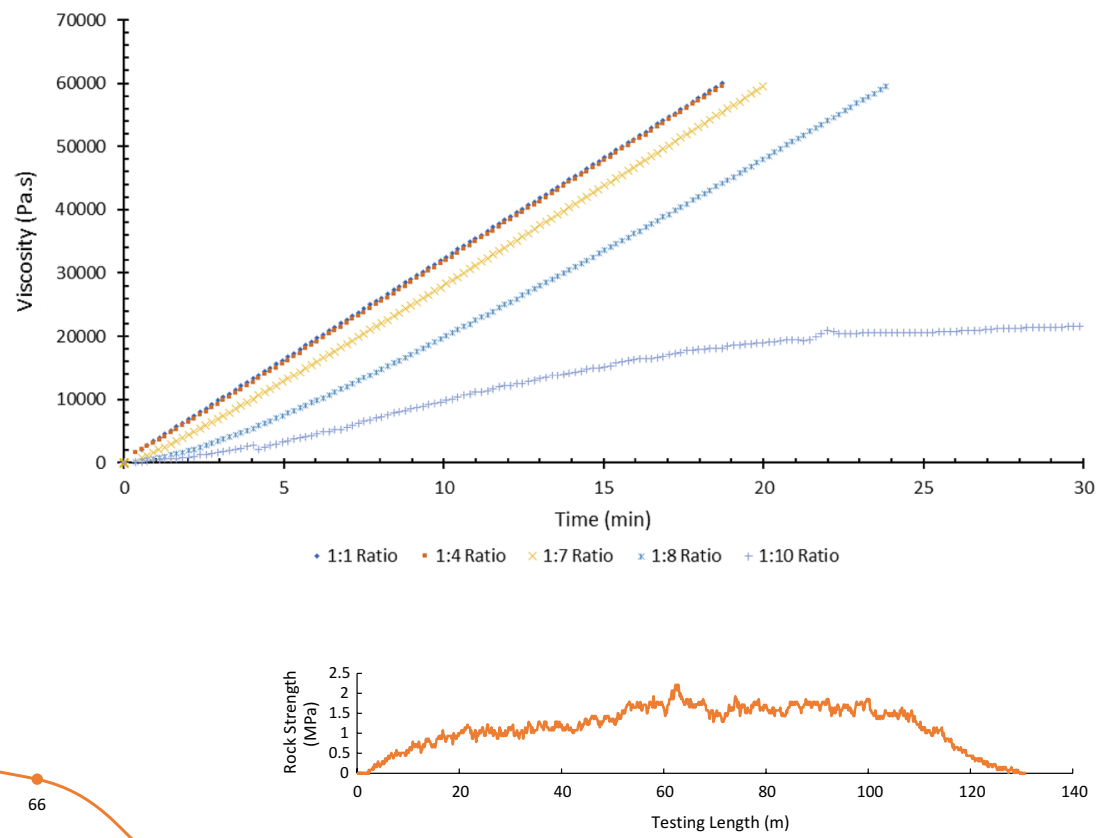

Fig. 13 Scratch test performed in $0.2 \mathrm{~mm}$ depth of cut

the repeat tests, it was found that room temperature tests were consistent. However, testing at higher temperature had a greater variation, which can be attributed to the poor heat convection of the highly viscous polymer (Fig. 12).

\section{Formation strengthening test}

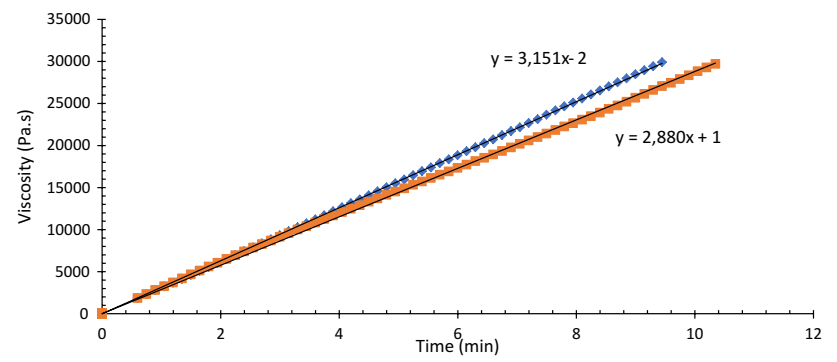

Fig. 12 Effect of temperature on polymers rheology

\section{Temperature effect on curing}

In Fig. 12, two tests are shown using the same volumes of polymer and water to observe the changes in rheology due to an increase in temperature. The blue line represents the testing performed at room temperature, and the orange line represents the testing conducted at a higher temperature $\left(60^{\circ} \mathrm{C}\right)$. It can be seen that an increase from room temperature $\left(25^{\circ} \mathrm{C}\right)$ to $60{ }^{\circ} \mathrm{C}$ decreased the rate that rheology increased over time. However, no changes in maximum rheology can be seen from these data. From
The formation strengthening supplied by the injection of the reactive fluid into an unconsolidated sand formation was recorded by completing a scratch test (Fig. 13). It was found that the polymer increased the rock strength by $1.5-2 \mathrm{MPa}$ which is a miniscule amount. The wellbore strengthening effect of the polymer may not be a key characteristic to justify its use in the field; however, strengthening it does supply some benefits when using it as LCMs in weak formations.

\section{Discussion}

\section{Permeability reduction}

The permeability reduction tests were completed under the condition of the polymer being injected into a watersaturated formation and hydrating with formation water only. The results presented on the polymer's effectiveness assumed that no contamination occurred to the polymer prior to reaching the formation. Contamination with water had a notable reduction in effectiveness as shown in Fig. 11. However, please note that significant contamination was necessary to greatly reduce the effectiveness. For this reason, a 
spacer is recommended for field applications of the polymer. From the preliminary testing completed, it was found that oil spacers were not a good option as a spacer fluid due to the density differences leading to the polymer displacing the oil. For this reason, it is advised that a wiper plug is used as a barrier for injection through the drill string. Results similar to those recorded in the experiments would be realistic with the correct application of the reactive polymer, i.e. correct injection method, large enough volume used, use of an effective spacer, etc.

\section{Rheology}

It was found that as the hydration ratio increased, the final viscosity of the reacted polymer continued to decrease. This shows the importance of developing an optimal methodology that minimises contaminating the polymer prior to entering the formation.

The results presented had limitations in finding the final maximum viscosity of low hydration ratios. However, repeat tests using equipment that can support higher shear stresses are not required, since the viscosity is high enough that any differences in maximum viscosity are negligible.

It was concluded that the reactive polymer's rheology at $60-70{ }^{\circ} \mathrm{C}$ did not significantly change, meaning that it is expected to have similar performance under higher temperature conditions. The rate of increase in viscosity decreased slightly; however, it is negligible since the rate is still very high. The experimental results confirm that no extra time is necessary for hydration under increased temperature conditions. Testing at higher temperatures was beyond the scope of this project.

\section{Formation strengthening}

The formation strengthening tests were performed by conducting a scratch test on unconsolidated sands that had been injected with the polymer. Due to very low strength of the formation and the stickiness of the polymer, it was difficult to prevent the build-up of sand and polymer on the PDC bit during the tests. This build-up leads to an increased resistance along the sample, thus leading to a high-strength reading. Another difficulty was achieving a homogenous sample with the polymer being evenly reacted and spread throughout the sample.

The scratch test data in this paper should only be used for an indication of the wellbore strengthening effect from the reactive polymer and not be an exact expected result. The polymer was tested under optimal conditions of temperature and hydration ratios, meaning that the results were recorded for a best-case scenario. The results do indicate that the polymer does supply a wellbore strengthening effect and may help support unconsolidated formations when encountered during drilling. This leads to the conclusion that the reactive polymer has added benefits over other LCMs when treating losses into unconsolidated formations. Since the strengthening effect recorded was quite small, even at optimal conditions, the effect will likely be negligible under borehole conditions.

\section{Conclusion}

- In this paper, a series of tests were performed using a fluid loss simulator to investigate a reactive polymers' effectiveness at reducing the permeability of high permeability formations. The results show that it effectively reduced the permeability of the unconsolidated sand formations tested.

- The rheology testing showed that using a hydration ratio between 1:1 and 1:6 would give the highest viscosity, thus the greatest resistance to being displaced. Increasing the hydration volume ratio to $\sim 1: 8$ began to reduce the maximum viscosity, with significant reductions occurring with even greater hydration ratios.

- The wellbore strengthening supplied by the reactive polymer was found to be miniscule under borehole conditions.

- It can be concluded that with an effective implementation, i.e. correct hydration time and hydration volumes, reactive polymers will effectively reduce the permeability of rock formations such as unconsolidated sandstones. However, further testing would be required to investigate the effectiveness of fractured formations.

- No conclusions were formed on the applicability of the reactive polymer, i.e. the pumpability, injecting in formation, erosion, etc. Further testing would be required to investigate these concepts.

Open Access This article is distributed under the terms of the Creative Commons Attribution 4.0 International License (http://creativeco mmons.org/licenses/by/4.0/), which permits unrestricted use, distribution, and reproduction in any medium, provided you give appropriate credit to the original author(s) and the source, provide a link to the Creative Commons license, and indicate if changes were made.

\section{References}

Abdelaal K, Ovrevik K, Knezevic R-N, Husodo R (2016) First successful implementation of an advanced fibrous organic based LCM and optimized casing design in Europe: a case history from drilling a 6022 m HPHT exploration well in Vienna Basin, Austria. Society of Petroleum Engineers, Dallas 
Al-saba Mortadha T, Nygaard R, Saasen A, Nes O-M (2014) Lost circulation materials capability of sealing wide fractures. Society of Petroleum Engineers, Dallas

Bugbee JM (1953) Lost circulation-a major problem in exploration and development. American Petroleum Institute, Washington

Caughron DE, Renfrow DK, Bruton JR, Ivan CD, Broussard PN, Bratton TR, Standifird WB (2002) Unique crosslinking pill in tandem with fracture prediction model cures circulation losses in deepwater Gulf of Mexico. Society of Petroleum Engineers, Dallas

Droger N et al (2014) Degradable fibre pill for lost circulation in fractured reservoir sections. Society of Petroleum Engineers, Dallas

El-Sayed M, Ezz A, Aziz M, Waheed SA (2007) Successes in curing massive lost circulation problems with a new expansive LCM. Society of Petroleum Engineers, Dallas

Hashmat MD, Sultan AS, Rahman S, Hussain SMS (2016) Crosslinked polymeric gels as loss circulation materials: an experimental study. Society of Petroleum Engineers, Dallas

Ivan C, Bruton J (2003) How can we best manage lost circulation? American Association of Drilling Engineers, Houston

Kumar A, Savari S, Jamison DE, Whitfill L (2011) Applications of fiber laden pill for controlling lost circulation in natural fractures. American Association of Drilling Engineers, Houston
Murchison WJ (2006) Lost circulation for the man on the rig. Murchison Drilling Schools, Inc, Albuquerque

Savari S, Kumar A, Whitfill DL, Miller M, Murphy RJ, Jamison DE (2013) Engineered LCM design yields novel activating material for potential application in severe lost circulation scenarios. Society of Petroleum Engineers, Dallas

Vidick B, Yearwood JA, Perthuis H (1988) How to solve lost circulation problems. Society of Petroleum Engineers, Dallas

Whitfill DL, Jamison DE, Wang M, Angove-Rogers A (2007) Preventing lost circulation requires planning ahead. Society of Petroleum Engineers, Dallas

Publisher's Note Springer Nature remains neutral with regard to jurisdictional claims in published maps and institutional affiliations. 